\title{
Can humanoid nurse robots replace human nurses?
}

\author{
Rozzano C. Locsin ${ }^{1 *}$ and Hirokazu Ito
}

*Correspondence: locsin@medsci.tokushima-u.ac.jp

1Professor of Nursing, Institute of Biomedical Sciences. Tokushima University Graduate School, Tokushima, Japan.

${ }^{2}$ Assistant Professor of Nursing, Institute of Biomedical Sciences, Tokushima University Graduate School, Tokushima, Japan.

\begin{abstract}
Background: The certainty of future technological developments in robotics and artificial intelligence can alter how nursing is practiced today and in the future. In 1992, Peck argued that while advancing technologies will take over the predictive and prescriptive health care practice, the future of nursing will rest on the irreplaceable human touch, specifically "Tender Loving Care."

Discussion: If nursing is the expression of TLC, and the science of caring supports this expression as nursing, is it true that contemporary nursing practice is the engagement of human beings with caring as its core? Is the practice of nursing grounded in caring? With recent advances in robot technologies and artificial super intelligence (ASI) will there be Humanoid Nurse Robots (HNRs) 'nurse' persons with TLC?

Conclusion: As fundamental to nursing practice grounded in the theory of Technological Competency as Caring in Nursing, the ultimate purpose of technological competency as caring in nursing is to know persons as caring who are participants in their care, rather than simply objects of care. The process of 'knowing persons as caring' in nursing is communicated as technological knowing, mutual designing and participative engaging.
\end{abstract}

Keywords: Technological Competency, caring, technology, theory, Humanoid Nurse Robots, nursing

\section{Introduction}

How is nursing practiced and who practices nursing? Answers to these essential questions are critical to understanding whether or not humanoid nurse robots can, will, or may replace human nurses in their practice. Nursing is a discipline of knowledge and a practice profession grounded in the ideal value of nursing as integral to human health care and well-being. Descriptions of nursing, definitions of what is a nurse, and the relationships within the nurse-nursed situations underscore the substantive focus of whether or not humanoid nurse robots (HNRs) can replace human nurses in practice.

The word nursing lends itself to a variety of definitions and descriptions. Its simple classification as a 'noun' does not provide significant insights other than that it is derived from the verb'nurse, nurses. 'With this in mind the American Nurses Association (n.d.) [1], defines nursing as the protection, promotion, and optimization of health and abilities of persons, in the prevention of illness and injury, facilitation of healing, alleviation of suffering through the diagnosis and treatment of human response, and advocacy in the care of individuals, families, groups, communities, and populations.
However, debates about nursing as a profession provide ways to clarify the needed appreciation of nursing as a discipline, in that it is a unique perspective, a distinct way of viewing all phenomena, which ultimately defines the limits and nature of its inquiry" [2, p. 115]. As such, while normative science is held as a standard dictating the progression of a discipline in the way that Kuhn [3] has acknowledged, to Shaw [4] such expectation may not reflect what nursing really is, furthering the idea that the status of the nursing discipline will continue to evolve indefinitely. With this indication, Perry [5] posited the quintessential statement about the discipline of nursing developing to the stage in which nurses do "think nursing."

Nonetheless, while the popular process guiding the practice of nursing today - as delineated by the American Nurses Association (n.d.) - is to follow a routine recipe often called the nursing process involving assessment, diagnosis, planning and outcomes, implementation and evaluation, such process is simply a guide leading nursing care activities towards predictable outcomes from planned and implemented nursing actions or interventions, as if human beings are predictable. Understood as an apprenticeship, nursing guided by this practice process 
Locsin et al. Journal of Nursing 2018,

recipe is dictated solely by prescribed procedures and by accomplishing nursing tasks.

The (un)certainty of the domain of nursing, i.e. considering the metaparadigms of nursing [6] as person, environment, health, and nursing, provide opportunities for nurse scholars to explore the meanings of the concepts central to nursing. Donaldson and Crowley [2] as well as Fawcett [6] have categorized the thematic boundaries of nursing practice; (1) laws and principles. While these themes provide boundaries for focus of the discipline regarding knowledge development, the $21^{\text {st }}$ century engagement with future technological developments in robotics and artificial intelligence is altering how nursing will be practiced. This exploration of the nursing domain of the future transcends what nursing practice will be.

In 1992, Peck [7] argued that while advancing technologies will take over predictive and prescriptive health care practice, the future of nursing will still require the irreplaceable human touch, specifically TLC or Tender Loving Care. If nursing is the expression of TLC, and the science of caring grounds this expression of nursing, the question is, does our contemporary nursing practice maintain the engagement of human beings interacting with caring as a core attribute? Is our practice of nursing grounded in caring? Locsin and Purnell [8] have proposed the Universal Technological Domain as encompassing the technological environment where nursing now and in the future will be recognized reflecting a realist perspective towards understanding the integral nature of nursing in fostering human health and well-being. Fundamental to human health, technologies in human care are the central characters in the unfolding uncertainty or certainty of future nursing practice engagements.

\section{Purposes}

This paper describes issues, views, and propositions about humanoid robots and their influences on the discipline and professional practice of nursing. In ilustrating 'knowing persons as caring'as the nursing process grounded in the Theory of Technological Competency as Caring in Nursing, $[9,10]$ the dynamic nursing process events of technological knowing mutual designing, and participative engaging, validate the impact and value of anthropomorphic intelligent machines on the development of a new ontology and epistemology of nursing practice and caring science in the technological world.

\section{Technology, robotics, and nursing}

As early as 1928, the mechanistic view of nursing practice was popular as completion of tasks, so that programming nursing chores provided the impetus for the consideration of a humanoid nurse. ERIC, the United Kingdom's first robot was manufactured, exhibited, toured, and displayed. However, Eric disappeared, was lost and vanished without a trace. Fortunately, the London Museum of Science was able to find blueprints, pictures and film clips, and from these evidences 'resurrected' it, and a ...new ERIC was manufactured. Today, it is part of the permanent display of the museum marking 500 years of Robotics [11].

Fast forward in the development of humanoid robots, in 2013 various types of technological advancements influenced health care, particularly robots with artificial general intelligence [12]. While these robots improved nursing time and nurse safety allowing more time for patient interactions, in 2015, a self-contained robot RIBA II [13] was fabricated and programmed to lift patients, although still automated to respond to human 'orders' with a much improved physicality creating a vision of likeness to human beings.

Today, the quest for real-life-like humanoid robots has been put to the test. While Masahiro Mori's [14] theory of the Uncanny Valley was influential in creating less human-like robotic physiognomies, contemporary roboticists and computer scientists are engaged in designing, developing, and manufacturing Humanoid Robots (HRs) which are in most sense, human-like. Not only is the physiognomy the target of roboticists, but the robots'functions as well. In 2016, researchers from Georgia Institute of Technology developed a robot that can now simulate sponging motions as if simulating a nurse performing bed baths using the traditional process [15]. However.....the question regarding robots being able to replace human nurses in their practice remains noteworthy.

Some 90 years after the first robot was designed, developed, and manufactured, Honda developed and used a seemingly autonomous robot called ASIMO@ [16], prepared to welcome visitors and provide timely responses to questions from prospective human clients. Today, possibilities continue and one can picture this situation:

A patient walks into the Emergency Department and sits in front of the "triage nurse," a computer that uses advanced algorithms to ask questions based on the patient's answers. Then a robot draws the patient's blood, and still another one computes real-time nurse schedules and bed availability to decide if the patient can be admitted. "Follow me," the robot says [17, p. 24]. In 2016, Mesquita, Zamarioli, and Carvalho [18] in their study of robots and patents used in nursing care practices, published in the Online Brazilian Journal of Nursing, found thirty five patents. $40 \%$ of these were related to the creation of robots to aid in the mobility of patients; $28.5 \%$ had to do with the development of robots to aid daily activities; $23 \%$ were about robots created for physiological assessment and monitoring rehabilitation. The reality of robot manufacturing now occupies the technological environment of healthcare offering a variety of robots that are to be used in the nursing care arena.

\section{What are anthropomorphic intelligent machines?} The following common terms unravel the similarities of names given to these robotic intelligent machines. Since Capek [19] published the play R.U.R. or Rossums Universal Robots, (the first time the word robot was used), varying definitions have surfaced in the lexicon of robotics and computer science giving legitimacy to several descriptions of the term based 
on functionalities, physiognomy, and artificial intelligence.

- Robots - machines controlled by computers that are used to perform jobs automatically [20]

- Humanoid / Android - A humanoid robot is a robot with its overall appearance based on the human body [21].

- Automaton - a machine that operates on its own without the need for human control, or a person who acts like a machine, without thinking or feeling [20].

- Intelligent machines - deep learning systems, the most advanced form of machine learning that is at the heart of recent breakthroughs in Al. It can match humans in recognizing images [22].

\section{Technology and nursing practice}

The contemporary practice of nursing is often differentiated as an independent or autonomous practice, or a process of nursing that is dependent on completion of nursing tasks dictated by planned, programmed, and specified interventions. With advances in nursing science, it is necessary for nurses to expand the understanding and application of practice to meet requisite technological demands for future human health care in settings in which futurist human persons may be the general population.

Perhaps as a consolation or a clarification of the looming influence of technologies in human care, Pols [23] claimed that there is no such thing as technology, "but that there are many technologies, each with their own characteristics and specificities. In grouping/lumping together of so many different objects into one, while expecting the same effect of all of them, is problematic" [p.2]. Future practices of nursing involving theory-based engagements and dictated by intelligent know-how without the prescriptive or predictive practice procedures that have identified contemporary nursing may predominate. Such imagery seems to answer current nursing practice questions about the nursing profession being deterred from engaging in innovative practices because of the limitations imposed by a dependent, procedure-based practice.

\section{Five dimensions of technologies}

In consideration of easily identifying technologies in human health care, five categories of dimensions are described:

- Technology as completer of human beings to re-formulate the ideal human being, such as replacement parts, either mechanical (prostheses) or organic (transplanted organs);

- Technology as machine technologies, e.g. computers and gadgets, enhancing nursing activities to provide quality patient care, such as Penelope or da Vinci in the operating theatres $[24,25]$

- Technologies that mimic human beings and human activities to meet the demands of nursing care practices, e.g. cyborgs (cybernetic organisms) or anthropomorphic machines and robots such as 'nursebots'or'robonurse' [26].

- Technology as enhancer of human qualities exemplified in the potentials of cybernetic organisms; and
- Technology as enhancer of human qualities of organic/ biological versions as chimeras.

\section{Humanoid robots and nursing}

Soon humanoid nurse robots (HNRs) fitted with Artificial Super Intelligence (ASI) [12] may be built that can compute much faster than human beings can do today. How can this be achieved? This will be possible with Technological Singularity, the future point in time when artificial intelligence will best human intelligence and be able to improve itself autonomously, as described by Kurzweil [27]. Will this even happen, and if so, can 2050 as proclaimed by Barrat [12] in his 2013 book Our Final Invention be the year?

Nevertheless, one should consider knowledge generation, data upsurge, and the capacity of technologies to capture and process, not only as linear, organized or constructed data. Watson $\odot$, a computer manufactured by IBM $\odot$ that is able to compete in speed-based $Q$ and $A$ activities is already beating human competitors in the game Jeopardy@ [28]. Cognitive systems in robot intelligence processes propel artificial super intelligence to newer heights. The future predictions of $\mathrm{Al}$ hinge in part on natural language processing (NLP) rather than structured and organized data alone.

Recently, revealed as the 'label' assigned to 'human beings who compute," "computers" were persons such as those employed by the National Aeronautics and Space Agency (NASA) during the 1950s. Computers were human beings as they did computations using their mental capacities and extraordinary talent.

The progress in computerization advanced in exponential ways from manual gadgetries, e.g. cash registers, to electronic machines, from analogue computers to digital ones, today, competing for supremacy is what is called the "quantum computer" [29]. With all the advances in computerization and robotic engineering, nursing as a profession needs to be on the front row thereby facilitating the rethinking of its current and future practice processes. If the professional practice of nursing is much like a prescription of tasks and expectations of cause and effect relationships, with all the technological and computer-based advances available today, robots can be programmed and will replace human nurses.

Can humanoid nurse robots replace human nurses?

The legitimacy of the uncertainty of whether or not humanoid nurse robots can replace human nurses depends upon the ontology and epistemology of nursing. Tanioka [30] has argued that while the possibilities of robot utility in healthcare is apparent in most health care institutions today, these technological machines (robots) can be limited simply to performing menial tasks like housekeeping chores or to the delivery of medications to human nurses. Equipped with ASI, in which computerization is beyond be the ultimate expression of task completion with seemingly intelligent machines. Consequent to the aforesaid perspectives is the awareness of human be- 
ings beyond being 'human,'and the post-human becoming a serious and enduring concern simply because organic human beings will eventually meet their death. Contemporary health care practices can provide opportunities for human beings to decide to live longer lives with biological parts replaced by bioengineered and/or artificial parts.

How will these post-human beings engage in a nursing relationship with human nurses who may also be cybernetic organisms (cyborgs) themselves, or perhaps as completely organic, biological human beings, rather than as highly sophisticated biomechano-electronic beings? To meet expectations beyond being human, equipping post-humans or Humanoid Robots with quantum computers may be the ultimate response to the demand for faster capabilities beyond what is now achievable for an intelligent robot.

\section{Outlooks regarding replacements of human nurses with HNRs}

It may be inevitable that in the following situations, conditions are established that are conducive to replacing human nurses with humanoid nurse robots.

- When nursing practice is defined as completion of tasks wherein nurses perform efficient work with excellence dictated by precision and speed. If these characteristics mark the nurse, then nurses can be easily replaced by HNRs. From a technological stance, healthcare robots will perform more precise and efficient 'nursing work.'

- When nursing is viewed as the relationship between the nurse and nursed in which engagements, caring between, and co-creation in the moment are characteristics for unpredictability in a non-theoretically based practice as expert nurses according to Benner's [31] viewpoint, demands for superintelligence beyond current human expectations is critical.

- Nursing practice as human-to-human and as human to human-like relations can now be revisited and reviewed, for both the nurse and nursed may be human beings with technologically-enhanced constituency, and/or may partly be composed of biomechanical parts through artificial organ replacements or organic transplantations.

Ethico-moral concerns with humanoid robots

Yet thoughtful concerns about HRs and Nursing practice raise the following questions: Will HRs become or be superiors to human nurses? Ought HRs with ASI participate in health care as overlords, supervising the activities of organic human beings? Furthermore, should HRs receive payment for services rendered? On the other end of the end of the spectrum, should human beings enslave the HRs? Will human nurses enslave HNRs?

With these concerns focused on the kinds of nursing practices, robot physiognomies, robot capabilities, and the seriousness of ethical relationships - enslavement of HRs or human superiority, one thing is certain: technologies are here to stay and will never go away. If nursing is to continue to 'serve'humanity and survive as a profession and a practice discipline, a redefinition of its ontology and epistemology needs to occur. What will this redefinition be, and what will it be like to practice nursing with or for HNRs or with patients who have technological enhancements?

Furthermore, if in the future nursing practice and nursing care is achieved, most likely with HNR functions and features transitioning through formulaic engagements, how will this unfold? The formulaic process is proposed to include procedure-based nursing practice, together with autonomous nursing practice processes, mutual engagements expressed by nurse-HNR competencies grounded in the Transactive Relationship Theory of Nursing (TRETON) [30] and the inclusion of theory-based nursing care practice of knowing persons as caring grounded in the theory of the Theory of Technological Competency as Caring in Nursing.

Nurses' work is based on ethics. It is dangerous for HNRs not to be able to recognize its "own-self" like human beings. Human nurses also care according to the legal limits of practice. How much work is allowed for HNR? If accidents happen with or are caused by HNRs, who is responsible for the accidents?Discussions on these ethico-moral aspects are necessary.In a survey conducted by Ito [32], the question asked was "Who should take responsibility if a Humanoid Robot Nurse made a mistake?"The answer that gained most of the highest score was the nurse. It was 'the nurse's responsibility' that obtained the highest score.

\section{The Theory of Technological Competency as Caring in} Nursing $[8,9,10]$

\section{Assumptions of the theory}

These five assumptions describe elements that structure the theory, galvanizing the concreteness of the relationship among technology, caring, and nursing.

- Persons are caring by virtue of their humanness [33]. In nursing, caring is the substantive focus of the discipline. It is not the act or emotion portrayed towards another person. It is the substance of the domain directing the integral nature of nursing as a discipline of knowledge. "Persons are caring" is studied as fundamental to the practice of nursing.

- The ideal of wholeness is a perspective of unity [34]. Persons are known as wholes in ways shaped by philosophical truths and realities and the recognition of completeness in their being is without reference to composition of parts. As such the nurse focuses on the person being nursed [33] rather than focusing on fixing the person or completing the person's lack or missing "parts."

- Knowing persons is a multidimensional process [9].The nurse and nursed engage in appreciating, celebrating, supporting, and affirming each other, while allowing for mutual recognition as dynamic participants in their care. Technologies of health and nursing are elements for 
caring [9]. Through their practice, these nurses can know persons more fully as active contributors in their care, rather than only as objects of care.

- Nursing as a discipline and a professional practice [33] provides the essential opportunity for engagement in the scholarshipof practice grounded in caring.

Knowing persons as caring: The dynamic nursing process $[8,35,36]$

Towards the understanding, affirming, celebrating, and supporting of persons as caring individuals in an ever-changing world, the following processes of "knowing persons as caring" are declared:

- Technological knowing is using technologies to know persons in a shared relationship expressed as appreciating the persons' humanness, participating in dynamic caring nursing relationships, and extending knowing of persons as participants in their care, instead of being simply objects of care.

- Mutual designing is theprocess in which both the nurse and the one nursed together create and plan the care from which an organized and conjointly rewarding nursing practice fosters, affirms, supports, and celebrates the desire of the nursed to live and grow in the meaningfulness of one's own life.

- Participative engaging is the simultaneous practice of relating with the other. Its critical feature is evident in the continuous and recursive knowing of persons as caring. To practice theory-based nursing, these three dynamic nursing processes simultaneously engage the nurse with the one nursed. The co-created moment [37] of the nurse and nursed, while seemingly linear, is appreciated and understood as occurring unpredictably in the moment. The randomness of human reactions and the continuous change occurring moment to moment enrich the value of technological encounters within the Universal Technological Domain[34].

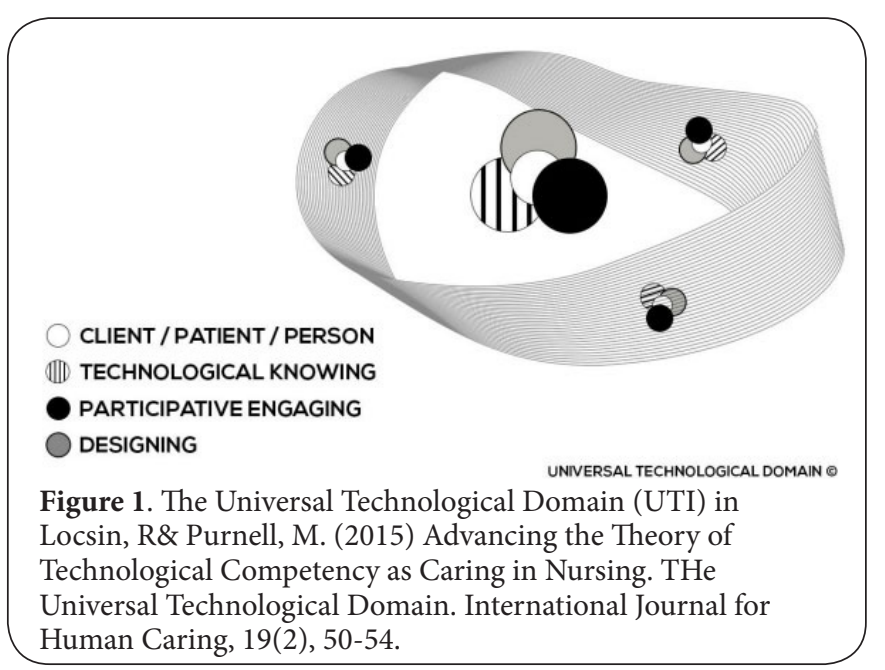

\section{Illustrating the dynamic nursing process}

Illustrated in the following story is the dynamic nursing process-a caring nursing situation that intently captured nursing as expressing knowing persons as caring involving technologies of human care.

One of my patients requested a new Intravenous line (IV) on her opposite arm, even though the one she had was safely infusing her IV fluids. I was extremely busy but I knew that her IV would not get changed until much later if at all, as shift change was occurring and she did not have veins that were easily accessed. I requested for the vein finder instrument and successfully inserted a new IV. My patient was so happy and told me that no one else had been able to "get a vein" on the first try. It seemed like a simpletask, but it made such a difference to her. I can appreciate that through competent use of the vein finder instrument, I was able to allow my patient to use her dominant hand instead of limiting her range of motion because of the IV location. She was ableto be more "whole" through the use of her dominant extremity.lt was so simple an act, but so moment-changing for her and also for me (Anonymous, n.d.).

In this nursing situation, technological knowing was affirmed as encompassing the expression of technological competency as caring in using the vein finder gadget; the mutual engagement was the shared lived experience between the nurse and nursed during the IV insertion -the reality of a technological encounter; and the participative engagement was the mutual appreciation within the co-created moment of the shared experience in a life-changing occasion.

\section{Conclusion}

Future technological nursing with humanoid nurse robots, the redefinition of the ontology (nature) and epistemology (practice) of nursing need to be re-envisioned and prioritized. If nursing is to continually'serve'humanity, it is anticipated that the science of caring be considered the main character in advancing, affirming, supporting, and legitimizing contemporary and future nursing practice of knowing persons as caring. The engagement of humans and human-like beings remains to be appreciated as the ultimate expression of technological competency as caring in nursing, in which knowing persons more fully as participants in their care is acclaimed best, rather than considering them as objects and recipients of care. Regardless, the critical question remains, will the practice of nursing while grounded in caring continue to epitomize the acceptability and justification of professional nursing as encounters of caring practice with intelligent machines?

How nurses practice nursing within the technological world of nursing, engaging humanoid robots as protagonists in the health care system needs to be re- envisioned. A practice that is grounded in contemporary nursing systems of prediction and prescription will serve HNRs best. In the future, such a practice will not serve the discipline and profession of nursing well, instead, it will serve task completion (if this continues to 
Locsin et al. Journal of Nursing 2018,

http://www.hoajonline.com/journals/pdf/2056-9157-5-1.pdf

doi: 10.7243/2056-9157-5-1

be nursing), instead of relating transactive relations between and among human beings and humanoid 'beings.'Are human nurses prepared and ready to emerge as technologically competent nurses who express their nursing as caring practice? In today's future will humanoid nurse robots replace human nurses?

Competing interests

The authors declare that they have no competing interests.

Authors' contributions

\begin{tabular}{|l|c|c|}
\hline Authors' contributions & RCL & HI \\
\hline Research concept and design & $\checkmark$ & $\checkmark$ \\
\hline Collection and/or assembly of data & $\checkmark$ & $\checkmark$ \\
\hline Data analysis and interpretation & $\checkmark$ & $\checkmark$ \\
\hline Writing the article & $\checkmark$ & $\checkmark$ \\
\hline Critical revision of the article & $\checkmark$ & $\checkmark$ \\
\hline Final approval of article & $\checkmark$ & $\checkmark$ \\
\hline Statistical analysis & $\checkmark$ & $\checkmark$ \\
\hline
\end{tabular}

Publication history

Editor: Pamela gail Hawranik, Athabasca University, Canada. Received: 13-Nov-2017 Final Revised: 03-Dec-2017

Accepted: 31-Dec-2017 Published: 11-Jan-2018

\section{References}

1. American Nurses Association (n.d.). What is nursing. I Website

2. Donaldson SK and Crowley DM. The discipline of nursing. Nurs Outlook. 1978; 26:113-20. | PubMed

3. Kuhn T. The structure of scientific revolution (2nd Ed.). Chicago: University of Chicago Press. 1970.

4. Shaw MC. The discipline of nursing: historical roots, current perspectives, future directions. J Adv Nurs. 1993; 18:1651-6. | Article | PubMed

5. Perry J. Has the discipline of nursing developed to the stage where nurses do 'think nursing'? J. of Adv Nurs. 10:31-37.

6. Fawcett $J$. The metaparadigm of nursing: present status and future refinements. Image J Nurs Sch. 1984; 16:84-9. | PubMed

7. Peck M.L. The future of nursing in a technological world. Hol. Nurs. Prac. 1992; 10:183-191.

8. Locsin R and Purnell M. Advancing the theory of Technological Competency as Caring in Nursing: The Universal Technological Domain. Int/ J. for Hum. Caring. 2015; 19:50-54.

9. Locsin R. Technological Competency as Caring in Nursing. Indianapolis, IN: Sigma Theta Tau International Press. 2005.

10. Locsin R. Technological Competency as Caring in Nursing (rev. Ed). Dumaguete City, Philippines: Silliman University Press. 2016.

11. [Science Museum]. Rebuild Eric: The UK's first robot. (2016, May 11). [Video File]. I Website

12. Barrat J. Our Final Invention. New York, NY: Thomas Dunne Books. 2013.

13. [ikinamo]. RIBA II care support robot for lifting patients(2011, October 24). Diginfo [Video File]. | Website

14. Mori M. The Uncanny Valley. Energy. 1970; 7:33-35.

15. King C.H, Chen T, Jain A and Kemp C.C. Towards an assistive robot that performs bed baths for patient hygiene. In IEEE/RSJ 2010 International Conference on Intelligent Robots and Systems at Taipei, Taiwan [Video File]. 2010; 319-324. | Website

16. [Yasser Alyasre]. ASIMO [Video File]. 2014. I Website

17. Glauser W. Artificial intelligence, automation and the future of nursing. Can Nurse. 2017; 113:24-6. | PubMed

18. Mesquita A.C, Zamarioli A.M and Carvalho E.C. The use of robots in nursing care practices: An exploratory-descriptive study. Online Brazilian Journal Nursing. 2016; 15:404-413.

19. Capek K. Rossum's Universal Robots (R.U.R). (Wyllie, Trans.).1921/2002Studio City, CA: Players Press.

20. Cambridge University Press. Cambridge dictionary [Online]. Cambridge, UK: Cambridge University Press. 2017. | Website

21. ScienceDaily. (n.d.). Humanoid robot. | Website

22. Financial Times. Intelligent machines are asked how their minds work. 2017. | Website

23. Pols J. Good relations with technology: Empirical ethics and aesthetics in robots. Nursing Philosophy. 2016; 18. | Article

24. Rojas P. The Penelope surgical instrument server makes its debut. | Website

25. Intuitive Surgical. Theda Vinci surgical system. 2017. | Website

26. Locsin $R$ and Barnard $A$. Technologicalcompetency as caring in cursing: A practice model. In A. Barnard \& R. Locsin, Technology and Nursing: Practice, Process, and Issues. London, UK: Palgrave-Macmillan. 2007.

27. Kurzweil R. The Singularity is Near: When Humans Transcend Biology. New York: Penguin Book. 2005.

28. [IBM Watson]. IBM Watson: How it works[Video File]. 2014. | Website

29. Savage N. Quantum computers compete for "supremacy." Scientific American. 2017. | Website

30. Tanioka T. The development of the transactive relationshiptheory of nursing. In Tanioka, T., Yasuhara, Y., Osaka, K, Ito, Hirokazu, and Locsin, R. Nursing Robots: Robotic Technology and Human Caring for the Elderly. Okayama, Japan: Fukuro Publishing. 2017.

31. Benner P. From Novice to Experts. MenloPark, CA: Addison-Wesley, Publishing. 1984.

32. Ito $\mathrm{H}$, Miyagawa $\mathrm{M}$, Kuwamura $\mathrm{Y}$, Yasuhara $\mathrm{Y}$, Tanioka $\mathrm{T}$ and Locsin $\mathrm{R}$. Professional nurses' attitudes towards the introduction of humanoid nursing robots (HNRs) in health care settings. J of Nurs and Healt Sci. 2015; 9:73-81.

33. Boykin A and Schoenhofer S. Nursing as Caring: A Model for Transforming Practice. Sudbury, CT. Jones and Bartlett. 2001.

34. Locsin RC. The Co-Existence of Technology and Caring in the Theory of Technological Competency as Caring in Nursing. J Med Invest. 2017; 64:160-164. | Article | PubMed

35. Locsin R. The theory of Technological Competency as Caring in Nursing: Guiding nursing and health care. Shikoku Acta Medica. 2016; 72:163170.

36. Locsin RC. The Co-Existence of Technology and Caring in the Theory of Technological Competency as Caring in Nursing. J Med Invest. 2017; 64:160-164. | Article | PubMed

37. Locsin R. Music as expression of nursing: A co-created moment. International Journal for Human Caring. 1998; 2:40-42.

Citation:

Locsin 1 RC and Ito H. Can humanoid nurse robots replace human nurses? Journal of Nursing. 2018; 5:1. http://www.hoajonline.com/nursing/2056-9157/5/1 